\title{
Acute Hepatitis A Infections among Veterans in Outbreak States, 2016-2018
}

\author{
Cynthia A. Lucero-Obusan ${ }^{1}$, Gina Oda $^{1}$, Patricia Schirmer ${ }^{1}$, Mark Holodniy ${ }^{1,} 2$ \\ ${ }^{1}$ Department of Veterans Affairs, Public Health Surveillance and Research, Palo Alto, California, United States, ${ }^{2}$ Stanford University, Department of \\ Infectious Diseases and Geographic Medicine, Stanford, California, United States
}

\section{Objective}

To conduct surveillance for acute Hepatitis A virus (HAV) infections in Veterans from states reporting outbreaks among high-risk individuals beginning in fiscal year (FY) 2017.

\section{Introduction}

Although cases of acute HAV have declined in recent years, elevated numbers of HAV infections began to be reported by California and Michigan in the fall of 2016 [1,2]. Since this time, associated outbreaks have been reported in 9 additional states (Arizona, Utah, Kentucky, Missouri, Tennessee, Indiana, Ohio, Arkansas, and West Virginia) [3]. No common source of food, beverages or drugs have been identified and transmission appears to be primarily person-to-person with high-risk individuals including people experiencing homelessness, those who use illicit drugs and their close direct contacts. In June 2018, CDC issued a Health Alert Network Advisory providing additional guidance on identification and prevention of HAV and updates on the outbreaks [4]. This prompted our office to more closely review our HAV surveillance, to identify Veterans who may be part of these outbreaks, and assess risk factors and outcomes of HAV infection.

\section{Methods}

We queried VA data sources starting in FY 2017 (October 1, 2016 - June 30, 2018) for HAV IgM laboratory tests and HAV-coded outpatient encounters and hospitalizations (ICD-10-CM: B15) to identify potential case patients. We performed a detailed chart review on all HAV IgM positive Veterans residing in or treated in an outbreak state during the identified outbreak time frame as reported by each state health department. Data elements collected included: (1) demographics; (2) risk factors, exposures and Hepatitis A vaccination status; (3) treatment locations (i.e. outpatient, Emergency Department, inpatient, intensive care unit); (4) presenting signs and symptoms; (5) laboratory data (including liver function tests (LFTs) and hepatitis testing); and (6) outcomes (i.e. deaths). County-level rates for positive HAV IgM test results were calculated using total unique users of VHA care for matching fiscal year time frames in each county as denominators.

\section{Results}

A total of 247 HAV IgM positive individuals were identified among 136,970 HAV IgM tests performed during the study period. Among these, 67 individuals resided in an outbreak state and were identified for further chart review. Additional laboratory review revealed that 5 of the 67 were positive for HAV Total Ab with no HAV IgM performed (all five patients came from a single facility and were asymptomatic at the time of testing). Based on review of clinical data for the remaining $62 \mathrm{HAV}$ IgM positive patients, $22(35 \%)$ did not meet the CSTE clinical case definition criteria [5] of having signs or symptoms consistent with acute viral hepatitis plus either jaundice or elevated ALT/AST levels. These patients were either asymptomatic or had relevant symptoms that could be explained by other diagnoses. None had documented jaundice and only 4 had any LFT elevation, which was mild (ALT: 60-83 IU/L, AST: 36-103 IU/L). There was often no mention of the positive HAV IgM test result in the patient visit records. In the cases where the results were documented, it was thought to be a false positive or cross reactivity, related to recent receipt of HAV vaccination, or prolonged persistence of HAV IgM from a prior infection. Patient characteristics of the 40 patients meeting the case definition are summarized in Table 1. None of confirmed cases had documentation of HAV vaccination prior to their acute infection. The top 5 counties of residence among confirmed cases were Jefferson, KY (7, 18\%), San Diego, CA (6, 15\%), Wayne, MO (4, $10 \%)$, Butler, MO $(3,8 \%)$ and Macomb, MI $(3,8 \%)$. Additionally, the top three counties (Jefferson, San Diego and Wayne) were each noted to have clustering of cases of acute HAV with risk factors of homelessness, substance abuse and/or needle exposure. Incidence rates for HAV IgM+ test results were calculated for all reported outbreak counties and the 25 counties with the highest rates are shown in Figure 1. 


\section{Conclusions}

Occurrence of acute HAV infections among Veterans during October 2016 - June 2018 followed patterns reported by states with outbreaks during the same time frame, including high hospitalization rates. Risk factors of homelessness, substance abuse and/or needle exposures were noted in the Veteran population, similar to national HAV outbreak data. County-level clustering of cases in states with outbreaks was also observed among Veterans, with incidence rates of HAV IgM+ as high as 13 per 10,000 Veterans. Additional education of VA providers is needed regarding recognition of and appropriate testing for acute HAV infections. HAV IgM should not be ordered in asymptomatic patients with normal LFTs as the pretest probability of HAV infection is low, leading to false positives and confusion in interpreting test results. Improving Hepatitis A vaccination rates among Veterans is important, particularly among individuals who are at increased risk for infection or complications from HAV and in outbreak states to limit further spread of this outbreak.

\section{References}

1. Hepatitis A Outbreak in California. Available at: <a href="https://www.cdph.ca.gov/Programs/CID/DCDC/Pages/Immunization/Hepatitis-AOutbreak.aspx">https://www.cdph.ca.gov/Programs/CID/DCDC/Pages/Immunization/Hepatitis-AOutbreak.aspx</a>. Accessed September 18, 2018.

2. Michigan Hepatitis A. Outbreak. Available at: <a href="https://www.michigan.gov/mdhhs/0,5885,7-33971550_2955_2976_82305_82310-447907--,00.html">https://www.michigan.gov/mdhhs/0,5885,7-33971550_2955_2976_82305_82310-447907--,00.html</a〉. Accessed September 18, 2018.

3. CDC. 2017 - Outbreaks of hepatitis A in multiple states among people who use drugs and/or people who are homeless. Available at: <a href="https://www.cdc.gov/hepatitis/outbreaks/2017MarchHepatitisA.htm">https://www.cdc.gov/hepatitis/outbreaks/2017March-HepatitisA.htm</a>. Accessed September 18, 2018.

4. CDC. Health Alert Network Advisory: Outbreak of Hepatitis A Virus (HAV) Infections among Persons Who Use Drugs and Persons Experiencing Homelessness. June 11, 2018. Available at: <a href="https://emergency.cdc.gov/han/han00412.asp">https://emergency.cdc.gov/han/han00412.asp</a> . Accessed September 18, 2018.

5. Position Statement CSTE. Hepatitis A, Acute 2012 Case Definition. Available at: <a href="https://wwwn.cdc.gov/nndss/conditions/hepatitis-a-acute/casedefinition/2012/">https://wwwn.cdc.gov/nndss/conditions/hepatitis-a-acute/case-definition/2012/</a>. Accessed September 18, 2018.

Table 1. Characteristics of Veterans with Acute HAV Infection, 2016-2018 


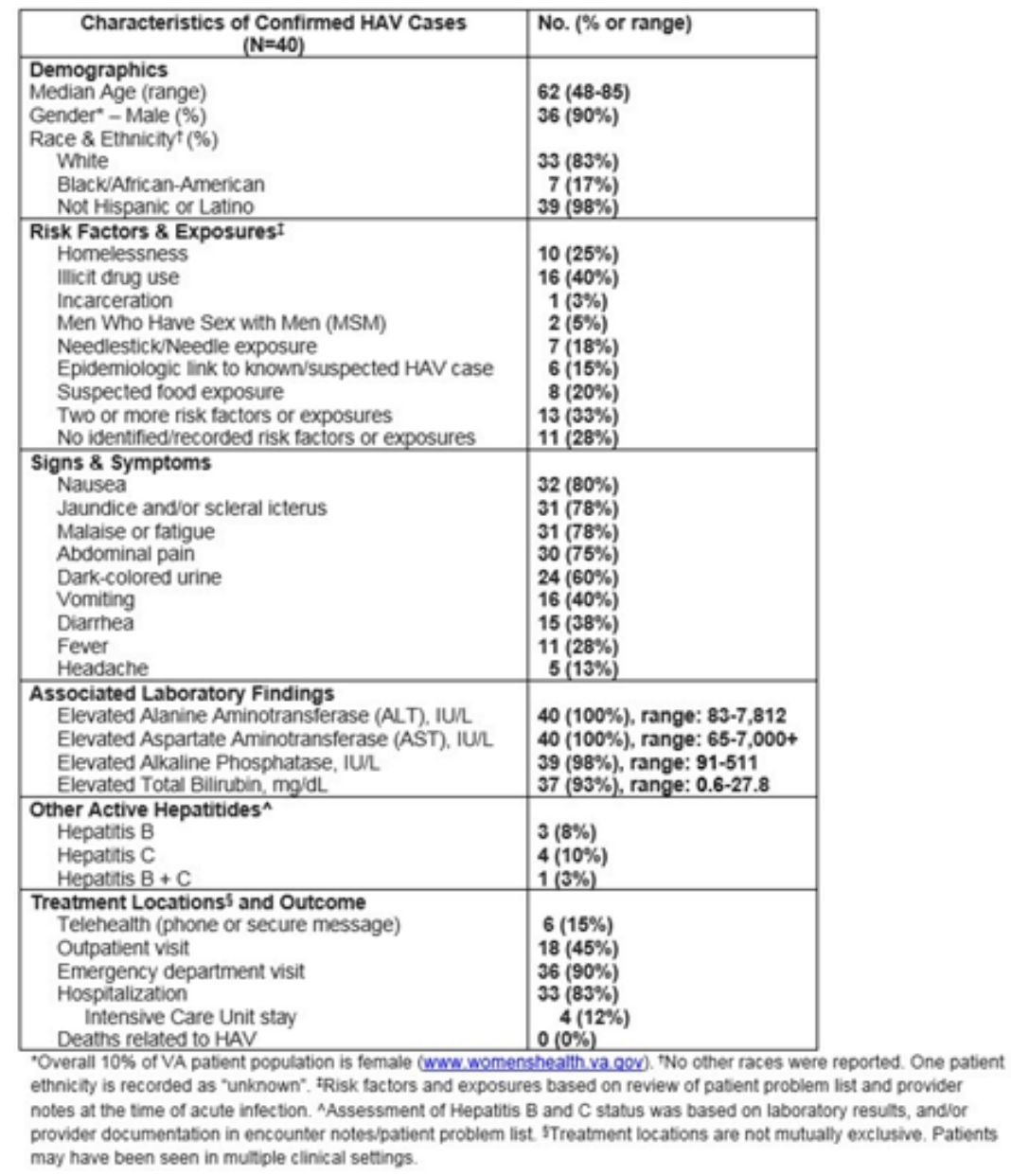

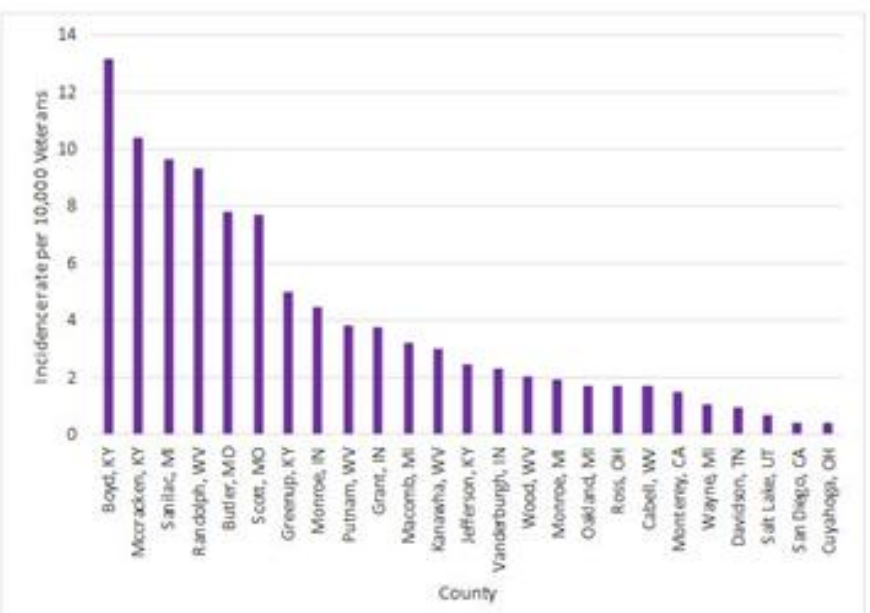

Figure 1. Reported Outbreak Counties with the highest HAV IgM+ Incidence Rates among Veterans, FY 2017 - FY 2018 